\title{
Content based Image Retrieval using Wavelet based Multi-resolution Analysis
}

\author{
Kalyanasundaram. K. \\ Assistant Professor \\ Department of Information Technology \\ Shri Angalamman College of Engineering \& \\ Technology, Tiruchirappalli 621105, Tamilnadu.
}

\author{
Sanoj. C.S. \\ Assistant Professor \\ Department of Information Technology \\ Shri Angalamman College of Engineering \& \\ Technology, Tiruchirappalli 621105, Tamilnadu.
}

\begin{abstract}
Content Based Image Retrieval (CBIR) is an emerging area of engineering application focusing on algorithms and methods to extract image features from a query image and retrieve similar images from large archives. It has found extensive application in medical imaging for both retrieval and automatic archiving. In this paper it is proposed to extract features using wavelet based multiresolution analysis and measure the classification accuracy of Naïve Bayes and K Nearest Neighbor ( K-NN) algorithm.
\end{abstract}

\section{Keywords}

Content Based Image Retrieval; Multiresolution; Haar Transform; Naïve Bayes; k-Nearest Neighbor

\section{INTRODUCTION}

Ever increasing amount of digitals medical images for diagnosis and treatment purposes, require efficient tools to effectively manage and utilize the information. Medical professionals need to retrieve medical images from the database for diagnosis, research, and teaching purposes. Retrieving on the basis of key words is not feasible as annotating huge amount of medical images is time-consuming and costly [1]. Content based image retrieval (CBIR) is adopted; this is an automatic system wherein images from a database are retrieved on the basis on image queries. Image retrieval based on image content similarity is more meaningful and reliable than text-based similarity. In CBIR, given an unknown query image, similar images with a known diagnosis are retrieved from the database, thus leading to more accurate diagnosis [2]. The features from the images are automatically extracted, classified and stored in the database; on query, feature from the query image is extracted and matched against those in the database. Matched images are retrieved by the CBIR system. A number of studies are available in literature [3-6] on medical image retrieval using CBIR.

The accuracy of a CBIR system depends on various factors like extraction of appropriate features from the image, classification of the image. The features extracted from an image are represented in the form of vectors. The retrieval accuracy, computational complexity, retrieval time depend on the dimension of the feature vector [7]. Higher the dimension of feature vectors, better the retrieval accuracy but the memory for storage, retrieval time and computational complexity increases. Thus is it important to find balance between the dimension of feature vectors and the accuracy with satisfactory storage and computational requirements. The database may contain images of similar kind but win different resolution, thus feature extraction cannot be efficiently done using the initial image resolution. This leads to deterioration of retrieval accuracy. To cope with this problem, multiresolution in wavelet transform domain is used. In multiresolution, the transforms decompose a signal into coarse space and many detail space called sub bands and are implemented by filter banks, through filtering and sampling [8]. Multiresolution analyses are used for image detection [9], texture analysis and classification [10] and image retrieval [11].

In most multiresolution analyses, pyramidal schemes are implemented. Gaussian and Laplacian filters are used to extract features. The Gaussian filter smoothens the images, thus reducing noise in the image and allows for simple processing. The space scale representation and pyramidal representation are the two different techniques used in Gaussian multiresolution.

In this paper it is proposed to extract the feature vectors using multiresolution Haar transforms[12]. The extracted features are classified using Naïve Bayes and k-Nearest-neighbor. This paper is organized into the following sections; section II contains related works in image retrieval using multiresolution analyses. Section III briefly introduces the proposed methodology and discusses the Haar transform, Naïve Bayes and k-Nearest neighbor. Section IV gives details about the experimental setup and section $\mathrm{V}$ devotes on results obtained.

\section{LITERATURE REVIEW}

Traina et al., [13] presented a MultiWaveMed system, for indexing and extracting medical images through comparison of texture features. The proposed system implemented both Daubechies and Gabor wavelets to extract feature. The features thus extracted were organized in feature vectors. Based on the feature vectors, the images were organized through access methods, which perform query-by-content operations over images. Euclidean metric function and normalized Euclidean function s were used to compare the images. Experiments were conducted using both color and texture features for image retrieval. Results obtained show that the time required for query answer was very short with high precision.

Wang et al., [14] proposed a multiresolution region-based searching system for pathology image libraries. In the proposed method a region matching metric, Integrated Region Matching, was used to reduce the effect of inaccurate segmentation. The images are indexed and matched using wavelets and IRM. Database containing 70,000 image fragments was used for evaluating the system. Experimental results showed that the proposed system achieved about $90 \%$ precision when examining the best 50 matches for each query and also it took about one second of CPU time to retrieve images as per query. 
Chun et al., [7] proposed a CBIR method based on multiresolution color and texture features. In the proposed method, color and texture are extracted using multi resolution wavelet domain and combined. Experimental results proved that the proposed method yields higher retrieval accuracy than conventional methods. The experiments also showed that the retrieval accuracy was high even with images of various resolutions.

Contassot-Vivier et al., [15] investigated different types of multiresolution methodology. The two main complementary steps, the bottom-up and top-down strategies of the multiresolution was discussed. The differences between the signal treatment and the graph based method were highlighted. Comparison of different techniques like the Gaussian and Laplacian, space scale and pyramidal representation of the multiresolution was studied in detail.

\section{METHODOLOGY}

\subsection{Haar Wavelet Transform}

Haar transforms was developed by Alfred Haar in 1909. Haar transforms are possibly the simplest wavelet basis consisting of square shaped functions []. It represents the functions in an ortho normal function basis as in Fourier analysis. The Haar transform, transforms signals from the space domain to a local frequency domain. A Haar wavelet decomposes an image using both low-pass filtering and high-pass filtering, working first on image columns and then on image rows. For a Haar wavelet, in general it is true that

$h_{\varphi}=\left[h_{\varphi}(0), h_{\varphi}(1)\right]=\left[\frac{1}{\sqrt{2}}, \frac{1}{\sqrt{2}}\right]$

Then,

$h_{\psi}(0)=(-1)^{0} h_{\varphi}(1-0)=h_{\varphi}(1)=\frac{1}{\sqrt{2}}$

$h_{\psi}(1)=(-1)^{1} h_{\varphi}(1-1)=-h_{\varphi}(0)=-\frac{1}{\sqrt{2}}$

The Haar wavelet is given in equation 3,

$\psi(x)= \begin{cases}1 & 0 \leq x<1 / 2 \\ -1 & 1 / 2 \leq x<1, \\ 0 & \text { elsewhere }\end{cases}$

Scaling function $\varphi(x)$ can be described as

$\varphi(x)= \begin{cases}1 & 0 \leq x<1 \\ 0 & \text { otherwise }\end{cases}$

Haar Transforms decomposes signals into an average (approximation) component and a detail (fluctuation)

component [anuj]. A signal with $2^{n}$ sample values, the first average sub signal $a^{1}=\left(a_{1}, a_{2}, \ldots a_{N / 2}\right)$ for a signal length of $\mathrm{N}$ is given as follows:

$$
a_{n}=\frac{y_{2 n-1}+y_{2 n}}{\sqrt{2}}, n=1,2, \ldots ., N / 2
$$

And the first detail subsignal $d^{1}=\left(d_{1}, d_{2}, \ldots ., d_{N / 2}\right)$ is given as:

$$
d_{n}=\frac{y_{2 n-1}-y_{2 n}}{\sqrt{2}}, n=1,2, \ldots ., N / 2
$$

The transform is applied to all rows of the matrix, the approximation parts of each row transform is arranged in the first $\mathrm{n}$ columns and the corresponding detail parts in the last $\mathrm{n}$ columns. The process is repeated till the resultant matrix is at first level. The resultant matrix has four piece with each piece of dimension (number of rows/2)X(number of columns/2) as shown in the matrix. And the each piece is termed $A, H, V$ and $D$. $A$ is the approximation area, $\mathrm{H}$ is the horizontal area, $\mathrm{V}$ is vertical area and $\mathrm{D}$ is diagonal area.

$$
\begin{aligned}
& M=\left[\begin{array}{lllll}
x_{11} & x_{12} & \vdots & x_{13} & x_{14} \\
x_{21} & x_{22} & \vdots & x_{23} & x_{24} \\
\cdots & \ldots & \vdots & \ldots & \ldots \\
x_{31} & x_{32} & \vdots & x_{33} & x_{34} \\
x_{41} & x_{42} & \vdots & x_{43} & x_{44}
\end{array}\right] \\
& A=\left(\begin{array}{ll}
x_{11} & x_{12} \\
x_{21} & x_{22}
\end{array}\right) H=\left(\begin{array}{ll}
x_{13} & x_{14} \\
x_{23} & x_{24}
\end{array}\right) \\
& V=\left(\begin{array}{ll}
x_{31} & x_{32} \\
x_{41} & x_{42}
\end{array}\right) D=\left(\begin{array}{ll}
x_{33} & x_{34} \\
x_{43} & x_{44}
\end{array}\right)
\end{aligned}
$$

Haar transforms are memory efficient, fast and simple. It is reversible without the edge effects, thus they are widely used in wavelet analysis.

\subsection{Naïve Bayes Classifier}

Learning is simplified in Naïve Bayes classifier as the features are assumed to be independent of the class [rish]. Inspite of the independence assumption, the naïve Bayes classifier is effective in practice. The Naive Bayes classification algorithm is a probabilistic classifier. It is based on the conditional probability stated as:

$P(D / a)=\frac{P(a / D) \cdot P(D)}{P(a)}$

for data point $a$ and class $D$. Bayesian classifiers assign the unknown instance to the most likely class based on its feature vectors. Similarly the probability of $a$ is estimated as follows:

$$
P(D / a)=P(D) \cdot \prod P\left(a_{j} / D\right)
$$

Conditional probabilities of each object in the predicted class are estimated during training using the training data set. The main advantage of Naïve Bayes classifier is that the parameter's mean and variance obtained from a small set of training data is sufficient for classification. Naïve Bayes 
provide good results and easy probabilistic interpretation of the results.

\subsection{K-Nearest Neighbor}

In k-nearest-neighbor the entire dataset is stored in the system's memory. When an unknown instance $\mathrm{x}$ is submitted for classification, the class is found either by finding the $\mathrm{k}$ instances in the dataset that are closest to $\mathrm{x}$ or the $\mathrm{k}$ instances vote to determine the class. The distance between the entities are calculated using various distance functions or formulas. The most common method of measuring distance is Euclidean. Euclidean squared, city-block, Hamming, Chebyshev are some distance measuring methods used in knearest-neighbor.
The Euclidean distance is calculated as:

$$
D(x, p)=\sqrt{(x-p)^{2}}
$$

where $\mathrm{x}$ is the unknown instance and $\mathrm{p}$ is an example in the dataset.

\section{RESULT AND DISCUSSION}

In this experiment 97 images consisting of three class labels $(44,39$ and 14 respectively) were used for analyzing the proposed method. Multi resolution analysis using Haar wavelet was used for feature extraction. The original images and the decomposed images are shown in Figure 1 and 2 respectively.

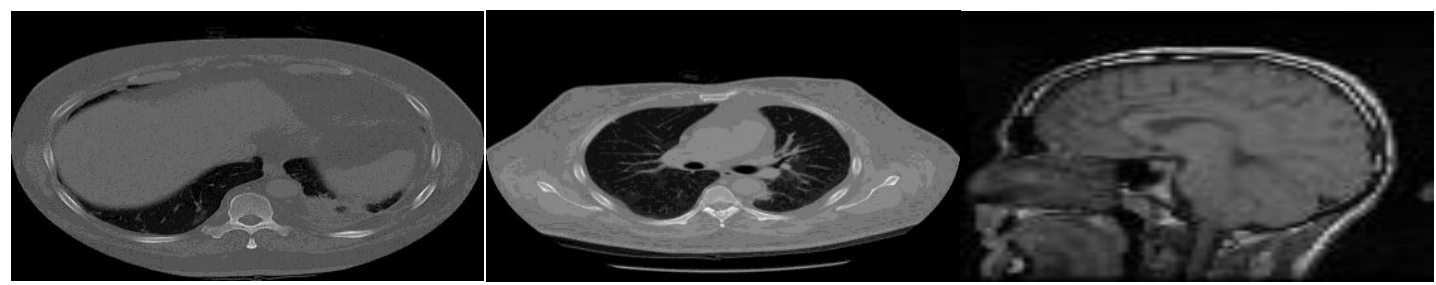

Figure 1: Sample images used in this work
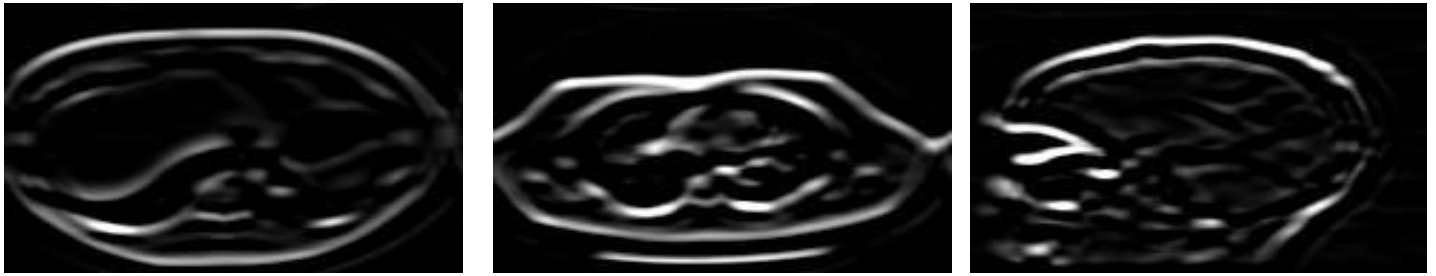

Figure 2: Obtained coefficients using multi resolution analysis

Using tenfold cross validation, the obtained coefficients were classified using Naïve Bayes and K Nearest Neighbor. The
Classification accuracy obtained in shown in figure 3. The misclassification by the classifiers is shown in Table 1 .

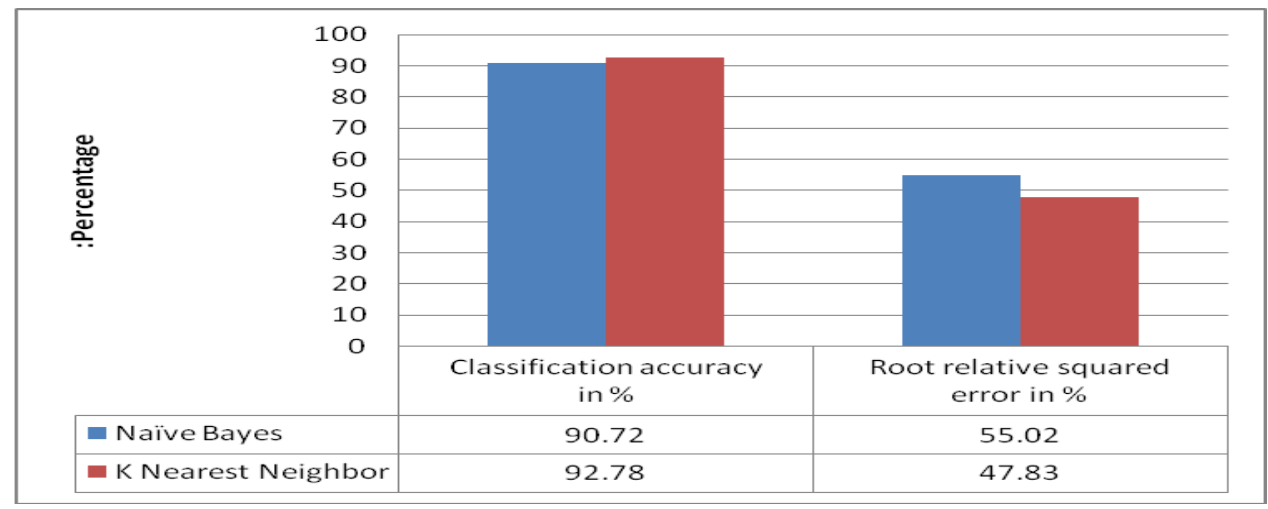

Figure 3: Classification accuracy and Root relative squared error 
Table 1: Misclassification By Naïve Bayes And K Nearest Neighbor

\begin{tabular}{|c|c|c|c|}
\hline Image No & Actual class & $\begin{array}{c}\text { Predicted class Naïve } \\
\text { Bayes }\end{array}$ & Predicted class K NN \\
\hline 10 & $\mathrm{c}$ & $\mathbf{a}$ & $\mathbf{a}$ \\
\hline 17 & $\mathrm{~b}$ & $\mathrm{~b}$ & $\mathbf{a}$ \\
\hline 20 & $\mathrm{c}$ & $\mathbf{a}$ & $\mathrm{c}$ \\
\hline 30 & $\mathrm{c}$ & $\mathbf{a}$ & $\mathrm{c}$ \\
\hline 47 & $\mathrm{~b}$ & $\mathbf{a}$ & $\mathrm{b}$ \\
\hline 49 & c & $\mathrm{c}$ & b \\
\hline 50 & $\mathrm{c}$ & $\mathrm{c}$ & $\mathbf{a}$ \\
\hline 59 & $\mathrm{c}$ & $\mathbf{a}$ & b \\
\hline 69 & $\mathrm{c}$ & $\mathrm{c}$ & b \\
\hline 70 & $\mathrm{c}$ & $\mathrm{c}$ & $\mathbf{b}$ \\
\hline 79 & $\mathrm{c}$ & $\mathbf{a}$ & $\mathrm{c}$ \\
\hline 97 & c & $\mathbf{a}$ & $\mathbf{b}$ \\
\hline
\end{tabular}

\section{CONCLUSION}

In this paper it was proposed to extract feature using multiresolution analysis using Haar Wavelet. Features extracted in this process was tested using Naïve Bayes and $\mathrm{K}$ Nearest Neighbor classifier. The classification accuracy from both the methods used was above $90 \%$. It was observed from the experimental results that larger number of images for training produced least classification errors. The misclassification for image of type 'a' with 44 samples did not produce any error, however image of type 'c' with only 14 samples contributed to $83.3 \%$ of the errors.

\section{REFERENCES}

[1] S. K. Chang, C. W. Yan, D. C. Dimitroff, and T. Arndt, "An intelligent image database system,"IEEE Trans. on Software Engineering, Vol.14, No.5, pp. 681-688, May 1988.

[2] A. M. Aisen, L. S. Broderick, H. Winer-Muram, C. E. Brodley, A. C. Kak, C. Pavlopoulou, J. Dy, C.-R.Shyu, A. Marchiori, "Automated storage and retrieval of thinsection CT images to assist diagnosis: system description and preliminary assessment", Radiology 228:265-270, 2003.

[3] K. Rajakumar, K. Rajakumar, "An Integrated Approach for Medical Image Retrieval Using PCA and Energy Efficient Wavelet Transform", European Journal of Scientific Research ISSN 1450-216X Vol.51 No.3 (2011), pp.340-348

[4] Avi Kak and Christina Pavlopoulou, "Content-Based Image Retrieval from Large Medical Databases." Proceedings of the First International Symposium on 3D
Data Processing Visualization and Transmission (3DPVT.02). IEEE 2002.

[5] T. Lehmann, M. G“uld, C. Thies, B. Fischer, K. Spitzer, D. Keysers, H. Ney, M. Kohnen, H. Schubert, and B. Wein. Content-based image retrieval in medical applications.

[6] Soo Beom Park, Jae Won Lee, Sang Kyoon Kim, "Content-based image classification using a neural network." Pattern Recognition Letter, Vol 25, Issue 3, Feb 2004, Pages 287-300.

[7] Young Deok Chun; Nam Chul Kim; Ick Hoon Jang; , "Content-Based Image Retrieval Using Multiresolution Color and Texture Features," Multimedia, IEEE Transactions on, vol.10, no.6, pp.1073-1084, Oct. 2008.

[8] A. Chebira, Y. Barbotin, C. Jackson, T. Merryman, G. Srinivasa, R. Murphy, and J. Kova“cevi'c, "A multiresolution approach to automated classification of protein subcellular location images," BMC Bioinformatics 8(210), 2007.

[9] S. Liu, C. F. Babbs, and E. J. Delp, "Multiresolution detection of speculated lesions in digital mammorgrams," IEEE Trans. Image Process., vol. 10, pp. 874-884, Jun. 2001.

[10] T. Chang and C. -C. J. Kuo, "Texture analysis and classification with tree-structured wavelet transform," IEEE Trans. Image Process., vol. 2, pp. 429-441, Oct. 1993. 
[11] S. Liapis and G. Tziritas, "Color and texture image retrieval using chromaticity histograms and wavelet frames," IEEE Trans. multimedia, vol. 6, pp. 676-686, Oct. 2004.

[12] Haar, Alfred (1910). "Zur Theorie der orthogonalen Funktionensysteme". Mathematische Annalen 69 (3): 331-371. doi:10.1007/BF01456326.

[13] Traina, A.J.M.; Castanon, C.A.B.; Traina, C., Jr.; "MultiWaveMed: a system for medical image retrieval through wavelets transformations," Computer-Based Medical Systems, 2003. Proceedings. 16th IEEE Symposium , vol., no., pp. 150- 155, 26-27 June 2003.

[14] Wang JZ. Pathfinder: multiresolution region-based searching of pathology images using IRM. Proc AMIA Symp 2000; 883-887.

[15] Sylvain Contassot-Vivier, Giosue Lo Bosco, Chanh Dao Nguyen. Multiresolution approach for image processing. 